\title{
Geometric and Grayscale Template Matching for Saudi Arabian Riyal Paper Currency Recognition
}

\author{
Suci Aulia', Bagus Budhi L ${ }^{2}$, Angga Rusdinar ${ }^{3}$, Yuyun Siti R ${ }^{4}$ \\ ${ }^{1,4}$ Department of Applied Science, Telkom University, Indonesia \\ ${ }^{2,3}$ Department of Electrical Engineering, Telkom University, Indonesia
}

\begin{tabular}{l} 
Article Info \\
\hline Article history: \\
Received Nov 28, 2017 \\
Revised Jul 9, 2018 \\
Accepted Aug 3, 2018 \\
\hline Keyword: \\
Banknote \\
Counterfeit \\
Geometric template matching \\
Grayscale template matching \\
PCR \\
SAR
\end{tabular}

Article Info

Article history

Received Nov 28, 2017

Revised Jul 9, 2018

Accepted Aug 3, 2018

\begin{abstract}
Detecting the authenticity of paper currencies using automated based Paper Currency Recognition (PCR) with image processing techniques was still a hot topic of discussion, due to the circulation of counterfeit currency that was still overwhelming in some countries. There was a downside along with this advancement in technology in the field of color printing, duplication, and scanning, because it was became one of the supporting factors of the increasing crime rate in production of counterfeit money. Our system has performed a PCR approach based on image processing techniques. In this study, the SAR banknote was the object to be recognized and detected its authenticity with the development of the previous method, which was incorporating the Geometric Template Matching and Grayscale Template Matching. In addition to the pattern recognition process, the classification process on $1 \mathrm{SAR}, 2 \mathrm{SAR}, 5 \mathrm{SAR}$, and $10 \mathrm{SAR}$ was also performed. From PCR test up to 100 sample data, for each tested banknote value obtained the average value of the best accuracy level from incorporating GeoMatchingScore and GrayMatchingScore for the classification process was $95.25 \%$. While the average level of system accuracy in recognizing counterfeit money on each banknote obtained a maximum value of $100 \%$.
\end{abstract}

Copyright $\odot 2018$ Institute of Advanced Engineering and Science. All rights reserved.

\section{Corresponding Author:}

Suci Aulia,

Department of Applied Science,

Telkom University,

Telekomunikasi Road, Dayeuh Kolot, Bandung, Jawa Barat 40257, Indonesia.

Email: sucia@tass.telkomuniversity.ac.id

\section{INTRODUCTION}

Counterfeiting has become the case in almost every country, thus motivating researchers to detect counterfeit currency based on image processing [1]-[4]. For example, especially in India counterfeiting has become a critical issue. Therefore, many researchers focus on detecting the authenticity of 100, 500, and 1000 rupees based on Neural Network [5]-[10] and other image processing based methods morphologically [11]-[18]. In addition to the Indian Rupee, other currencies are widely studied, including Euro [19]-[20], US Dollar [21]-[23], Saudi Arabian Riyal [22], Indonesian Rupiah [24] and Japan Yen [25]. One study of the currency recognition based on image processing was a study that has been done by Sawant [26]. In this study, the currency used was an Indian currency with an accuracy level approaching $90 \%$ based on 4 parameters, namely Dominant Color, Aspect Ratio, Mark ID and Latent Image. Similar research was still about the Indian currency recognition, which used the DWT algorithm for feature extraction and classification based on Probabilistic Neural Network (PNN) [27].

The result obtained by approaching both methods was quite good that was with the accuracy of 90.38\%. In addition to the Indian currency recognition, which has done a lot because of its high counterfeiting crime rates, currency recognition on dollars also widely popular among researchers as studied by F. Takeda [25]. In his journal, it was proposed a new technique to conduct a paper currency recognition 
and classification on Japan Yen and US Dollar, which was using neural networks. The NN structure coupled with the random mask method shows its effectiveness for processing data viewed from time and frequency domain. In addition to Indian Rupee and US dollar, Paper Currency Recognition (PCR) on Saudi Arabian Riyal based on the correlation between images has also been done. The method used for the classification was Radial Basis Function Network and the average accuracy level obtained was quite satisfactory that was 91.51\% [28]. Other research this paper referred to was PCR and classification on five currencies at once, including US Dollar (USD), Australian Dollar (AUD), Saudi Arabian Riyal (SAR), Euro (EUR), and Indian Rupee (INR) [22]. In this research, pattern recognition in Region of Interest (ROI) using neural network, while the process of classification using template matching method. The system has successfully recognized 5 INR, 20 INR, 20 Euro, 50 Euro, 20 AUD and 50 AUD banknotes. Pattern recognition on 1 SAR and 5 SAR banknotes are still in process, while pattern recognition on USD banknotes was failed.

Based on some of the studies presented above, PCR is still a challenging topic to study. Therefore, in this research we will study the currency recognition technique and its classification using template matching method, because based on research [22] it has successfully recognized five currencies namely USD, AUD, INR, EUR and SAR. In the previous study [28], PCR and classification of USD based on Canny Edge Detection and Template Matching obtained an average accuracy level of $95.625 \%$. In this study, the SAR banknote is the object to be recognized and detected its authenticity with the development of the previous method, which is incorporating the Geometric Template Matching and Grayscale Template Matching.

\section{RESEARCH METHOD}

In this study, the results of edge detection using canny edge detection obtained satisfactory results as recommended by [29]-[30] it seems to produce false detection in noisy environment. Based on other references [31], canny edge detection in pre-processing gives a fairly good classification result.The next step for image smoothening process in this research is done with two approaches, which are median filter and gaussian filter. Median filtering is a non-linear digital filtering technique which is often used to reduce and even eliminate noise [29]. Median filtering is often used in digital image processing because of its superiority on maintaining an edge value during noise removal process [32]. Figure 1 shows an illustration of the median filtering.

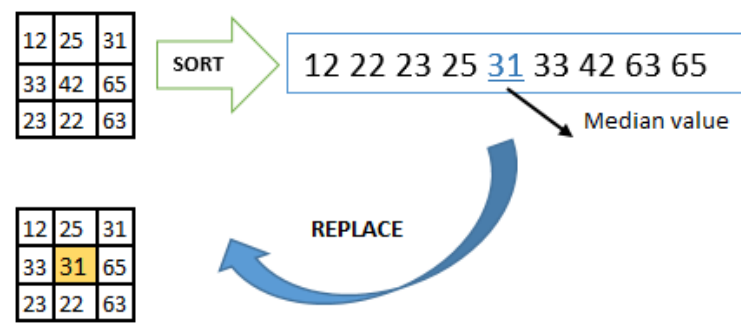

Figure 1. Input and output illustration of median filter without changing the edge pixel value

Based on Figure 1 above, median value replaced the center of median filter matrix based on equation (1) [33]-[35] :

$$
y[m, n]=\operatorname{median}\{x,[i, j],(i, j) \in w\}
$$

Where $w$ represents a neighbourhood centered on location. Another approach was gaussian filter, it was a smoothing technique on edge detection process [24]. The equation for edge matching algorithm of the image [36] is shown on equation (2):

$$
\log (x, y)=\frac{-1}{\pi \sigma^{4}}\left[1-\frac{x^{2}+y^{2}}{2 \sigma^{2}}\right] e^{-\frac{x^{2}+y^{2}}{2 \sigma^{2}}}
$$

Where $(x, y)$ represents the position of each pixels of the image and $\sigma$ was the Standard Gaussian Deviation. In image processing, Gaussian filter used was a two dimensional Gaussian filter. Therefore, each pixel direction has one dimensional Gaussian equation as follows: 


$$
G(x, y)=\frac{1}{\sqrt{2 \pi \sigma^{2}}} e^{\frac{-x^{2}}{2 \sigma^{2}}}
$$

Where $\sigma$ represented the standard deviation of value distribution. The distribution was assumed to have an average value equal to 0. The illustration of Gaussian distribution is shown on Figure 2 and Figure 3.

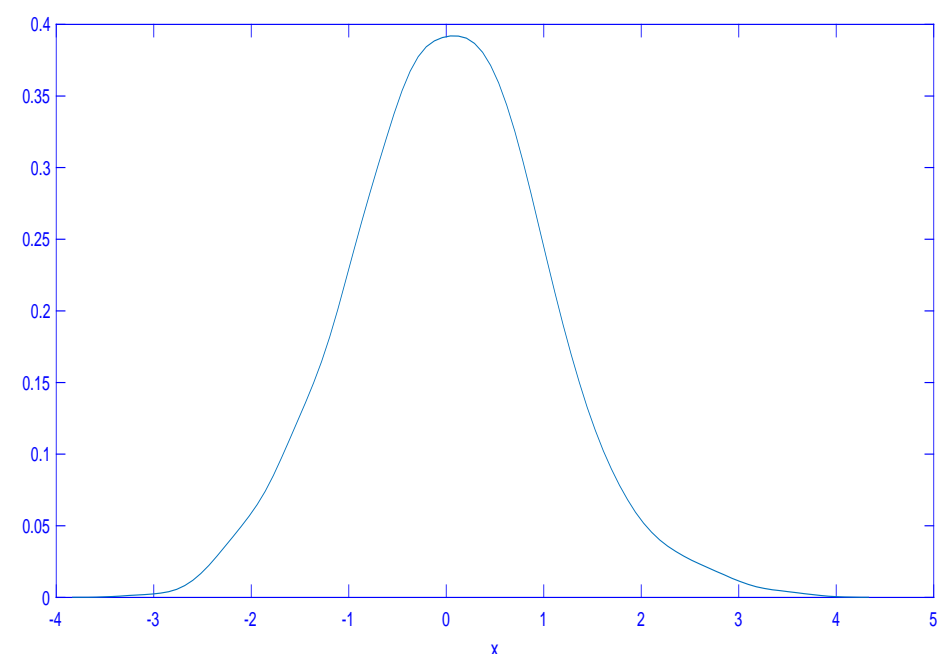

Figure 2. Value distribution graph of Gaussian 1D

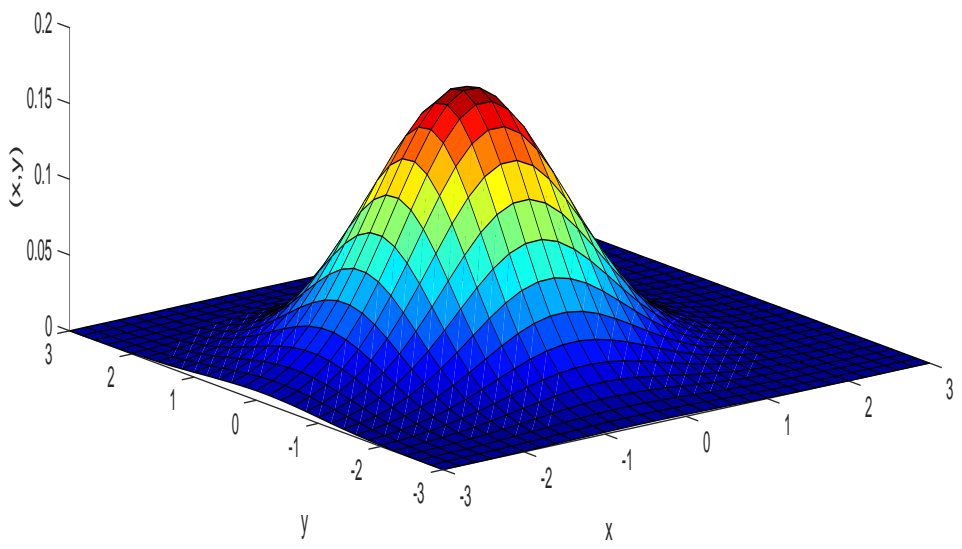

Figure 3. Value distribution graph of Gaussian 2D

The second step in this research after the smoothing process was parameter optimization. Template Matching was a matching technique used in the study, template matching was often accurate by combining geometric score and grayscale score parameters. Ghazi [37] has tested the accuracy level of Geometric Template Matching (GeTeM) to detect the Dinar currency for 100 times and it has the accuracy level of $91 \%$. GeTeM works by comparing this x value: $x=\left\langle x_{i}\right\rangle_{i=1}^{n}$ with this x' value: $x^{\prime}=\left\langle x^{\prime}{ }_{i}\right\rangle_{i=1}^{n}$ in time domain [38]. In his paper [38], Frank mentions GeTeM needs to be considered a powerful addition to the suite of tools that a time series analyst has at their disposal for the next future work, so that in this study trials were combined between GeTeM and Grayscale Template Matching (GrayTeM) for feature extraction process. The equation of the match score itself is shown by the following equation (4) [36]:

$$
\text { match } \text { score }_{\text {out }}=\frac{\text { matched pixels }}{\text { total pixels in ROI }} \times 1000
$$




\section{RESULTS AND ANALYSIS}

The system consisted of 3 main parts, namely 1) mechanical system of the scanner box the size of $18 \mathrm{~cm} \times 10 \mathrm{~cm} \times 15 \mathrm{~cm}$ to take the image of the banknote with a lighting from 4 led pieces and 180 degrees of light distribution, 2) web camera and PC that serves as the image processing system, and 3) actuator controllers which controls by assigning serial values (serial out of PC) using arduino UNO and DC motor to pull the banknote after being scanned with a webcam. The three systems are connected serially to the computer as a communication center, either as a system's database server, or as an existing actuator controller.

\subsection{System Testing Scenario}

To found out the best parameters that was used to test the authenticity of the banknote (PCR and its classification), the first step was to match the filter median variable $\mathrm{X}$ size $(1,5,10,15$, and 20) and Y size $(1,5,10,15$, and 20) each combination and Gaussian filter with divider parameters $(\mathrm{d} 5, \mathrm{~d} 10$, and $\mathrm{d} 15)$ on a number of training set of template images. After testing on the training set data, then the output was in the form of Geometric Matching Score (GeoMS) and Grayscale Matching Score (GrayMS). The values of GeoMS and GrayMS are analyzed and used as the threshold value for each banknote 1 SAR, 5 SAR, 10 $\mathrm{SAR}$, and $50 \mathrm{SAR}$. From the results of the training set data test, table $\mathrm{X}$ and table $\mathrm{Y}$ each shows GeoMS and GrayMS data for PCR 1 SAR. The data were taken based on the lowest score for every 10 attempts. Based on Table 1, the threshold value of GeoMS PCR 1 SAR taken from the lowest value on the top edge column was 901,488. Similarly, the threshold value of GrayMS PCR 1 SAR based on Table 2 was 858,782. Furthermore, after GeoMS and GrayMS were obtained for each SAR PCR, a test for the classification process was conducted.

Table 1. Geometry Matching Score of PCR 1 SAR

\begin{tabular}{cccc}
\hline $\begin{array}{c}\text { Number of } \\
\text { Test }\end{array}$ & Min Value & $\begin{array}{c}\text { Max } \\
\text { Value }\end{array}$ & Top Edge \\
\hline 10 & 823.837 & 901.988 & 901.488 \\
20 & 902.184 & 908.172 & 907.672 \\
30 & 909.341 & 913.821 & 913.321 \\
40 & 913.889 & 919.059 & 918.559 \\
50 & 919.536 & 921.716 & 921.216 \\
60 & 922.004 & 925.7 & 925.2 \\
70 & 926.04 & 930.747 & 930.247 \\
80 & 931.152 & 934.558 & 934.058 \\
90 & 935.195 & 942.113 & 941.613 \\
100 & 942.192 & 959.826 & 959.326 \\
\hline
\end{tabular}

Table 2. Grayscale Matching Score of PCR 1 SAR

\begin{tabular}{cccc}
\hline $\begin{array}{c}\text { Number of } \\
\text { Test }\end{array}$ & Min Value & Max Value & Top Edge \\
\hline 10 & 819.712 & 859.282 & 858.782 \\
20 & 859.339 & 859.833 & 859.333 \\
30 & 859.874 & 860.377 & 859.877 \\
40 & 860.401 & 860.798 & 860.298 \\
50 & 860.803 & 861.044 & 860.544 \\
60 & 861.076 & 861.429 & 860.929 \\
70 & 861.456 & 861.883 & 861.383 \\
80 & 861.965 & 862.317 & 861.817 \\
90 & 862.332 & 862.822 & 862.322 \\
100 & 862.848 & 864.557 & 864.057 \\
\hline
\end{tabular}

\subsection{Experimental Results}

The process of image acquisition was done by capturing the banknote coming into the scanner box with the lighting conditions of 4 LEDs arranged into $2 \times 2$ LEDs. From the capture result, the system then detected the value of the number with the largest font size and then cropped it as seen in Figure 4 and Figure 5. Figure 4 represents the Geometric Template Matching for each 1 SAR, 5 SAR, 10 SAR, and 50 SAR banknote. As for Grayscale Template Matching, it is represented in Figure 5.

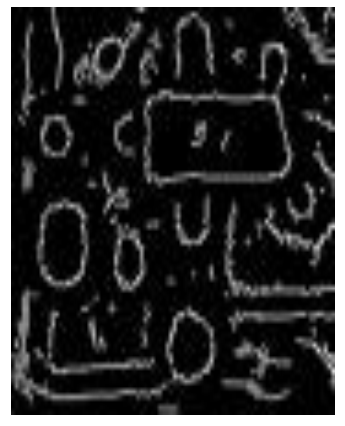

(a)

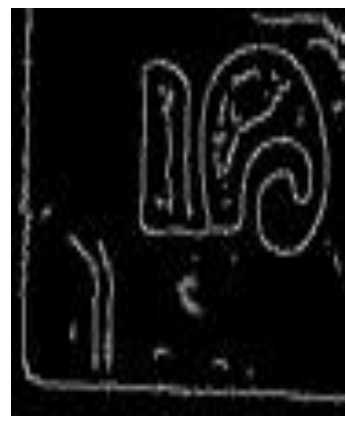

(b)

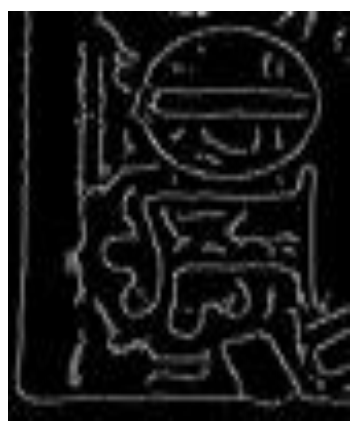

(c)

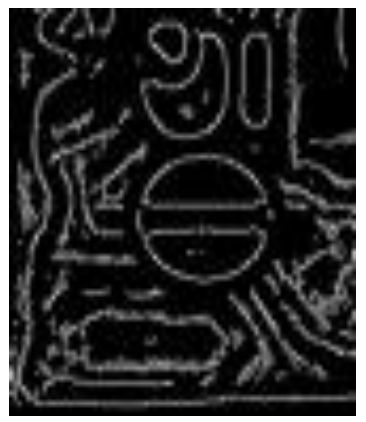

(d)

Figure 4. Image template geometric matching (a) $1 \mathrm{SAR}$, (b) $5 \mathrm{SAR}$, (c) $10 \mathrm{SAR}$, (d) $50 \mathrm{SAR}$ 


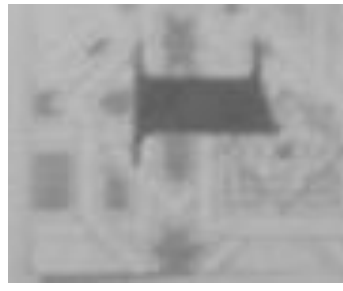

(a)

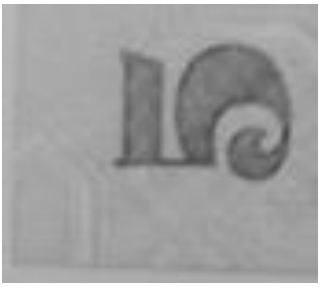

(b)

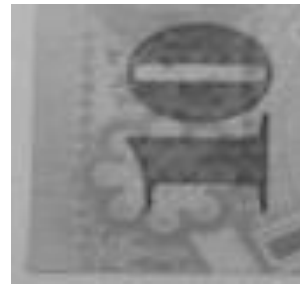

(c)

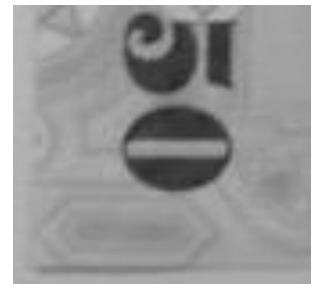

(d)

Figure 5. Image template grayscale matching. (a) $1 \mathrm{SAR}$, (b) $5 \mathrm{SAR}$, (c) $10 \mathrm{SAR}$, (d) $50 \mathrm{SAR}$

Parameters used as a reference in this study include median filter parameters, divider parameters on Gaussian filter, GeoMS and GrayMS. The median filter parameter which repeatedly modified was the value of X, Y. Figure 6 shows one of the min-max value search results from GeoMS for PCR 5 SAR from 100 experiments by testing each size of the median filter parameters and Gaussian divider parameters. The same is shown in Figure 7 for min-max values of GrayMS PCR 1 SAR.

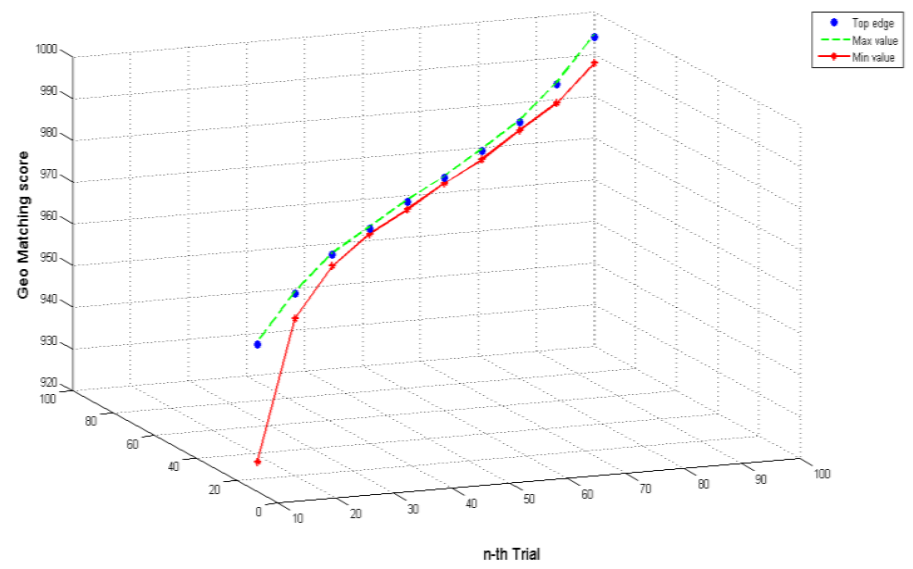

Figure 6. Geometric Matching Score graph for PCR 5 SAR

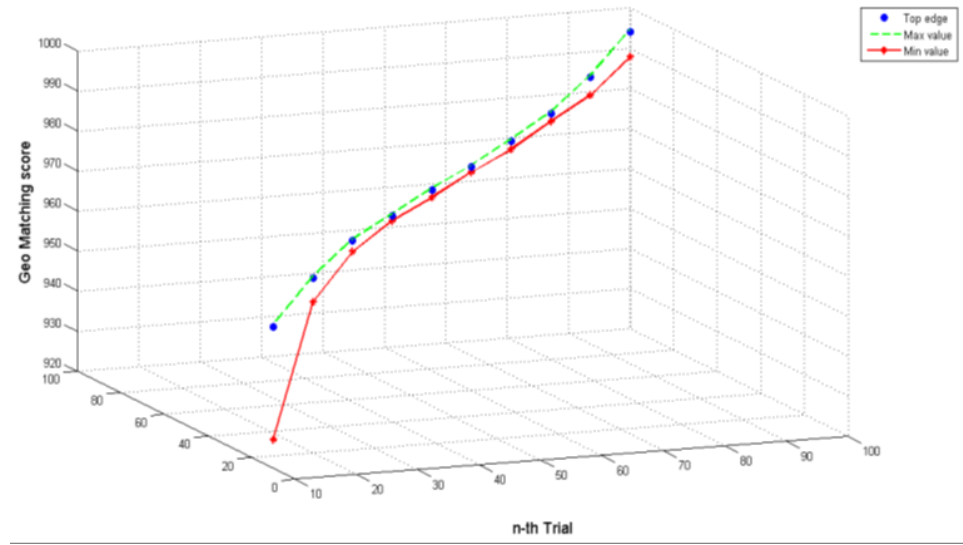

Figure 7. Grayscale Matching Score graph for PCR 1 SAR

Figure 7 represents min-max value for GeoMS PCR 5 SAR is 927.12 - 994.713, while from Figure 8 min-max value for GrayMS PCR 1 SAR is 941.292 - 959.796. The matching score values are stored as database which are then used as reference scores when performing PCR testing or classification. Based on 
the test results of 100 training data set, the comparison of score value obtained for each GeoMS and GrayMS templates on each banknote is shown in Figure 8, Figure 9, and Figure 10.

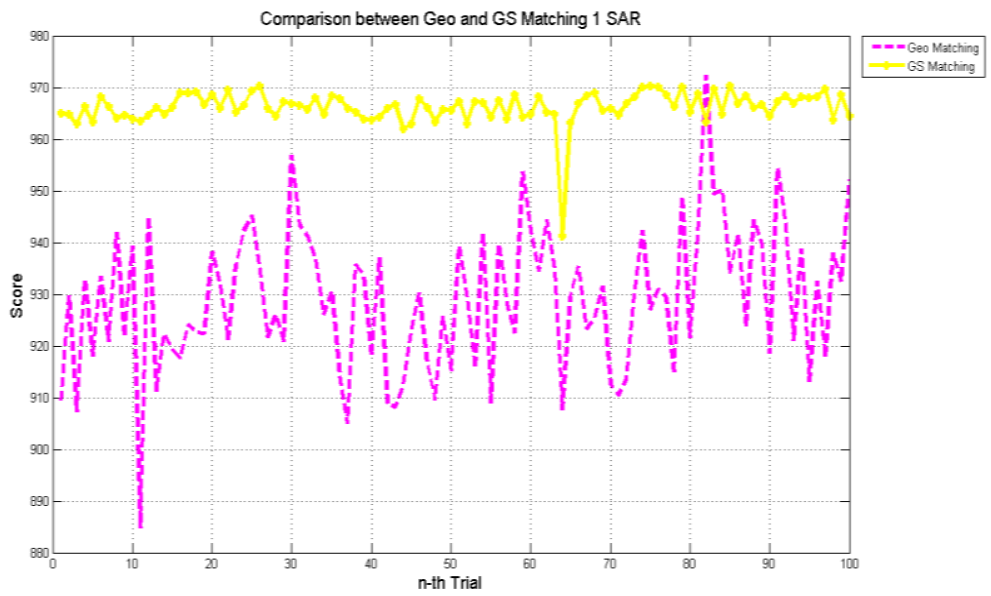

Figure 8. Comparison of GeoMS and GrayMS on PCR 1 SAR

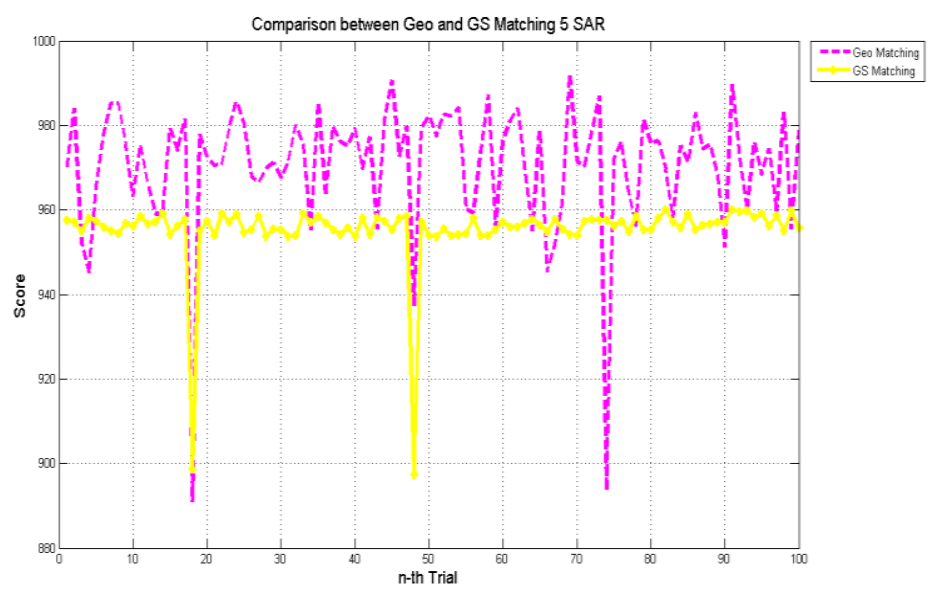

Figure 9. Comparison of GeoMS and GrayMS on PCR 5 SAR

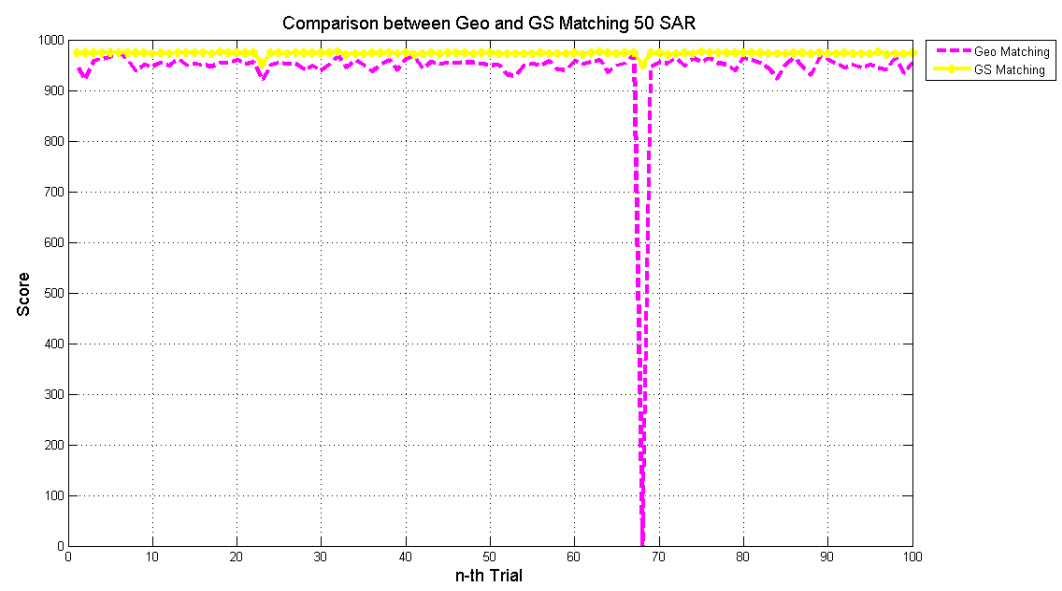

Figure 10. Comparison of GeoMS and GrayMS on PCR 10 SAR 


\begin{tabular}{cccccc}
\multicolumn{5}{c}{ Table 3.Accuracy Level of Currency Recognition and its Classification } \\
\hline No & Banknotes & True Positive & False Negative & Accuracy Level & $\begin{array}{c}\text { Success rate of detecting } \\
\text { counterfeit money }\end{array}$ \\
\hline 1. & 1 SAR & 99 & 1 & $99 \%$ & $100 \%$ \\
2. & 5 SAR & 86 & 14 & $86 \%$ & $100 \%$ \\
3. & 10 SAR & 97 & 3 & $97 \%$ & $100 \%$ \\
4. & 50 SAR & 99 & 1 & $99 \%$ & $100 \%$ \\
\hline
\end{tabular}

From PCR test to 100 sample data for each banknote value, obtained a result as shown in Table 3. From Table 3 obtained the average value of accuracy level of combining Geometric Template Matching and Grayscale Template Matching for the classification process was 95.25\%. While the average value of system accuracy level in the recognition of counterfeit money on each banknote (1 SAR, 5 SAR, 10 SAR, and 50 SAR) obtained a maximum value of $100 \%$.

\section{CONCLUSION}

In this paper, we have successfully demonstrated a PCR (Paper Currency Recognition) and its classifications test on Saudi Arabian Riyal 1 SAR, 5 SAR, 10 SAR, and 50 SAR with proposed techniques by combining Geometric Template Matching and Grayscale Template Matching methods to produce a quite satisfying result in counterfeit money recognition and the classification of its own currency. In the process of recognizing counterfeit money on each banknote system can detect $100 \%$, while the system performance for PCR classification with the best parameters of combining GeoMS and GrayMS reached 95.25\% positive recognition rate and $4.75 \%$ negative recognition rate.

\section{ACKNOWLEDGEMENTS}

This work has been supported by Internal Fund Research 2016 I from Telkom University.

\section{REFERENCES}

[1] T. Chakraborty, et al., "Review of Various Image Processing Techniques for Currency Note Authentication," Int. J. Comput. Eng. Res. Trends, vol/issue: 3(3), pp. 119-122, 2016.

[2] O. R. Devi, et al., "Survey on Paper Currency Recognition System," Int. J. Emerg. Trends Technol. Comput. Sci., vol/issue: 5(2), pp. 105-108, 2016.

[3] P. S. Nila, et al., "Review on Counterfeit Indian Paper Currency Recognition System," Int. J. Sci. Res. Comput. Sci. Eng. Inf. Technol., vol/issue: 2(2), pp. 88-91, 2017.

[4] B. Sharma, "Recognition of Indian Paper Currency based on LBP," Int. J. Comput. Appl., vol/issue: 59(1), pp. 2427, 2012.

[5] E. Pilania and B. Arora, "Recognition of Fake Currency Based on Security Thread Feature of Currency," Int. J. Eng. Comput. Sci., vol/issue: 5(17136), pp. 17136-17140, 2016.

[6] K. Chakraborty, et al., "Recent Developments in Paper Currency recognition System," Int. J. Res. Eng. Technol., vol/issue: 2(11), pp. 222-226, 2013.

[7] V. K. Jain, "Indian Currency Denomination Identification Using Image Processing Technique," Int. J. Comput. Sci. Inf. Technol., vol/issue: 4(1), pp. 126-128, 2013.

[8] P. J. Grace and D. Ph, "A Survey on Fake Indian Paper Currency Identification System," Int. J. Adv. Res. Comput. Sci. Softw. Eng., vol/issue: 6(7), pp. 340-345, 2016.

[9] R. Rathee, "A Review Paper on Currency Recognition System," J. Netw. Commun. Emerg. Technol., vol/issue: 6(8), pp. 5-6, 2016.

[10] S. Singh, et al., "Currency Recognition on Mobile Phones," 2014 22nd International Conference on Pattern Recognition IEEE, pp. 2661-2666, 2014.

[11] M. S. V. Vashishtha, "A paper currency recognition system using image processing to improve the reliability with pca method," Int. J. Eng. Sci. Res. Technol., vol/issue: 9655(6), pp. 172-175, 2015.

[12] P. D. Pawar and S. B. Kale, "Recognition of Indian Currency Note Based on HSV Parameters," Int. J. Sci. Res., vol/issue: 3(6), pp. 132-137, 2014.

[13] S. E. Ali and S. Mukherjee, "Challenges In Indian Currency Denomination Recognition \& Authentication," Int. J. Res. Eng. Technol., vol/issue: 3(11), pp. 477-483, 2014.

[14] Rashmi C. and D. H. K., "Image Processing Approach for INR Currency Note Number Recognition System for Automated Teller Machines," Int. J. Comput. Appl. Technol. Res., vol/issue: 5(8), pp. 539-542, 2016.

[15] I. A. Suresh, "Indian Currency Recognition and Verification Using Image Processing," Int. Res. J. Eng. Technol., vol/issue: 3(6), pp. 87-91, 2016.

[16] S. Sahu and T. Verma, "Identification of Paper Currency Techniques: A Survey," Int. J. Sci. Technol. Eng., vol/issue: 2(12), pp. 607-612, 2016. 
[17] B. S. Prasanthi and D. R. Setty, "Indian Paper Currency Authentication System using Image processing," Int. J. Sci. Res. Eng. Technol. (IJSRET), vol/issue: 4(9), pp. 973-981, 2015.

[18] H. Hassanpour, et al., "Feature extraction for paper currency recognition," 2007 9th International Symposium on Signal Processing and Its Applications IEEE, pp. 1-4, 2007.

[19] D. P. Universit, et al., "Mathematical and Statistical Methods for Actuarial Sciences and Finance," Milano, Springer Milan, 2010.

[20] F. M. Hasanuzzaman, et al., "Robust and Effective Component-Based Banknote Recognition for the Blind," IEEE Transactions On Systems, Man, And Cybernetics, vol/issue: 42(6), pp. 1021-1030, 2012.

[21] C. Bhurke, et al., "Currency Recognition Using Image Processing," Int. J. Innov. Res. Comput. Commun. Eng., pp. 4418-4422, 2015.

[22] J. W. Lee, et al., "A Survey on Banknote Recognition Methods by Various Sensors," Sensors, vol/issue: 17(313), pp. 1-34, 2017.

[23] M. Akbar, et al., "Original and Counterfeit Money Detection Based on Edge Detection," 2013 Int. Conf. Instrumentation, Commun. Inf. Technol. Biomed. Eng., 2013.

[24] F. Takeda and S. Omatu, "High Speed Paper Currency," IEEE transactions on neural network, vol/issue: 6(1), 1995.

[25] K. Sawant and C. More, "Currency Recognition Using Image Processing and Minimum Distance Classifier Technique," Int. J. Adv. Eng. Res. Sci., vol/issue: 6495(9), pp. 1-8, 2016.

[26] S. S. Sannakki and P. J. Gunjale, "Recognition and Classification of Currency Notes using Discrete Wavelet Transform,” Int. J. Emerg. Technol. Adv. Eng., vol/issue: 4(7), pp. 253-257, 2014.

[27] M. Sarfraz, "An intelligent paper currency recognition system," Procedia - Procedia Comput. Sci., vol. 65, pp. 538-545, 2015.

[28] B. B. Laksono, et al., "Operasi Valas : Identifikasi Nominal Dengan Metode Canny Edge Detection dan Template Matching Foreign Exchange : Nominal Identification Using Canny Edge Detection and Template Matching Abstrak Diskusi Sharpening dan Smoothing Sharpening merupakan penajama,” SNIKO, pp. 10-11, 2015.

[29] M. Krishnaveni, et al., "Improved Canny Edges Using Cellular Based Particle Swarm Optimization Technique for Tamil Sign Digital Images,” Int. J. Electr. Comput. Eng., vol/issue: 6(5), pp. 2158, 2016.

[30] R. Supriyanti, et al., "Brightness and Contrast Modification in Ultrasonography Images Using Edge Detection Results," TELKOMNIKA Telecommunication Comput. Electron. Control, vol/issue: 14(3), pp. 1090-1098, 2016.

[31] A. Fahrurozi, et al., "Wood Classification Based on Edge Detections and Texture Features Selection," vol/issue: 6(5), pp. 2167-2175, 2016.

[32] W. K. Pratt, "Digital Image Processing," vol/issue: 5(11), 2001.

[33] S. Shrestha, "Image Denoising Using New Adaptive Based Median Filter," Signal Image Process. An Int. J., vol/issue: 5(4), pp. 1-13, 2014.

[34] S. Surya and G. Thailambal, "Comparative Study on Currency Recognition System Using Image Processing," Int. J. Eng. Comput. Sci., vol/issue: 3(8), pp. 7723-7726, 2014.

[35] S. Chandel and M. Tyagi, "Evaluate and Propose a Novel Technique to Check Genuineness of the Currency Using Image Processing," Int. J. Comput. Sci. Trends Technol., vol/issue: 5(1), pp. 111-116, 2017.

[36] G. Sharma, et al., "Image Recognition System using Geometric Matching and Contour Detection," Int. J. Comput. Appl., vol/issue: 51(17), pp. 48-53, 2012.

[37] G. I. Raho, et al., "Cash Currencies Recognition Using k-Nearest Neighbor Classifier," Int. J. Web Semant. Technol., vol/issue: 6(4), pp. 11-21, 2015.

[38] J. Frank, et al., "Time Series Analysis Using Geometric Template Matching," IEEE Trans. Pattern Anal. Mach. Intell., vol/issue: 35(3), pp. 1, 2012.

\section{BIOGRAPHIES OF AUTHORS}

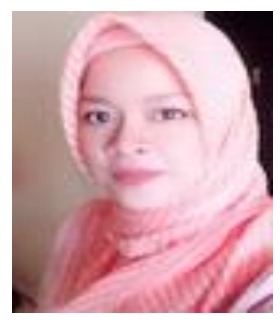

Suci Aulia, M.T. is an lecturer at Telkom University, Departement of Applied Science. She is concern in signal processing since 6 years ago. She has published several papers in her research especially image signal processing fields such as her latest publication under the title "Hog And Ica Based Face Recognition System On A Surveillance Video”, etc.

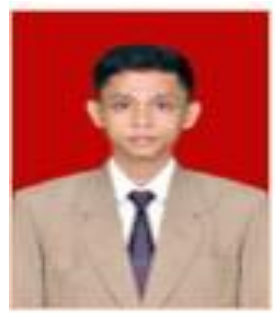

He graduated from Electrical Engineering at Telkom University Year 2015 with the title Skipsi "Automatic Money Changer: Identification Currency Input And Operating Operation Of Valas Exchange With Canny Edge Detection And Template Matching Method ". During the study he was active as a researcher at Digital Control Laboratory. 


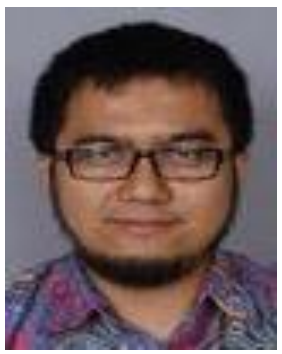

Angga Rusdinar,Ph.D is an lecturer at Telkom University, Departement of Electrical Engineering. His skills and ecxpertise focus on Pattern Recognition, Computer Vision, Robotics Electronic Engineering, and Control Systems Engineering.

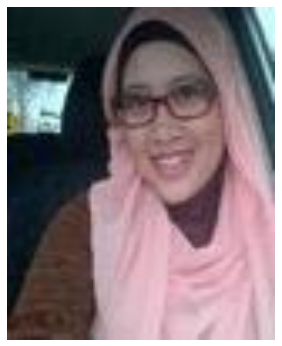

Yuyun siti rohmah ST,.MT is an lecture at Telkom University. She is concern in transmission telecommunication system. She has published paper about performance of Orthogonal Frequency Division Multiplexing and Orthogonal Wavelet Division Multiplexing. 